\title{
Phonon scattering in graphene over substrate steps
}

\section{Sevincli, Haldun; Brandbyge, Mads}

\section{Published in:}

Applied Physics Letters

Link to article, DOI:

$10.1063 / 1.4898066$

Publication date:

2014

\section{Document Version}

Publisher's PDF, also known as Version of record

Link back to DTU Orbit

Citation (APA):

Sevincli, H., \& Brandbyge, M. (2014). Phonon scattering in graphene over substrate steps. Applied Physics Letters, 105(15), 153108. https://doi.org/10.1063/1.4898066

\section{General rights}

Copyright and moral rights for the publications made accessible in the public portal are retained by the authors and/or other copyright owners and it is a condition of accessing publications that users recognise and abide by the legal requirements associated with these rights.

- Users may download and print one copy of any publication from the public portal for the purpose of private study or research.

- You may not further distribute the material or use it for any profit-making activity or commercial gain

- You may freely distribute the URL identifying the publication in the public portal

If you believe that this document breaches copyright please contact us providing details, and we will remove access to the work immediately and investigate your claim. 


\section{AIP/Apolied Physics Letters}

\section{Phonon scattering in graphene over substrate steps}

H. Sevinçli and M. Brandbyge

Citation: Applied Physics Letters 105, 153108 (2014); doi: 10.1063/1.4898066

View online: http://dx.doi.org/10.1063/1.4898066

View Table of Contents: http://scitation.aip.org/content/aip/journal/apl/105/15?ver=pdfcov

Published by the AIP Publishing

\section{Articles you may be interested in}

Resonant carrier-phonon scattering in graphene under Landau quantization

Appl. Phys. Lett. 103, 253117 (2013); 10.1063/1.4852635

Electronic and phonon bandstructures of pristine few layer and metal doped graphene using first principles calculations

AlP Advances 3, 032117 (2013); 10.1063/1.4794949

Low-temperature anomalies in the thermal conductivity of plastically deformed crystals caused by phonon-kink scattering

Low Temp. Phys. 38, 1055 (2012); 10.1063/1.4765094

Wave packet simulations of phonon boundary scattering at graphene edges

J. Appl. Phys. 112, 024328 (2012); 10.1063/1.4740065

Hydrogen vibrational modes on graphene and relaxation of the $\mathrm{C}-\mathrm{H}$ stretch excitation from first-principles calculations

J. Chem. Phys. 133, 054505 (2010); 10.1063/1.3474806

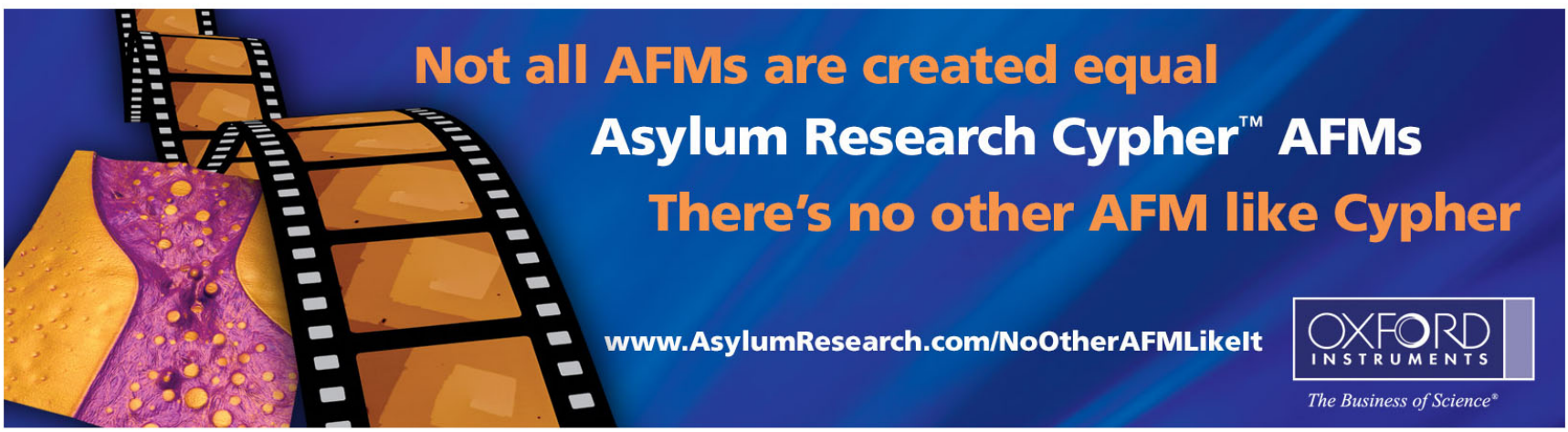




\title{
Phonon scattering in graphene over substrate steps
}

\author{
H. Sevinçli ${ }^{1,2, a)}$ and M. Brandbyge ${ }^{2,3, b)}$ \\ ${ }^{1}$ Department of Materials Science and Engineering, Izmir Institute of Technology, Gülbahçe Kampüsü, \\ 35430 Urla, Izmir, Turkey \\ ${ }^{2}$ Department of Micro- and Nano-technology (DTU Nanotech), Technical University of Denmark, \\ DK-2800 Kongens Lyngby, Denmark \\ ${ }^{3}$ Center for Nanostructured Graphene $(C N G)$, Department of Micro- and Nano-technology, \\ Technical University of Denmark, DK-2800 Kongens Lyngby, Denmark
}

(Received 11 September 2014; accepted 2 October 2014; published online 15 October 2014)

\begin{abstract}
We calculate the effect on phonon transport of substrate-induced bends in graphene. We consider bending induced by an abrupt kink in the substrate, and provide results for different step-heights and substrate interaction strengths. We find that individual substrate steps reduce thermal conductance in the range between $5 \%$ and $47 \%$. We also consider the transmission across linear kinks formed by adsorption of atomic hydrogen at the bends and find that individual kinks suppress thermal conduction substantially, especially at high temperatures. Our analysis show that substrate irregularities can be detrimental for thermal conduction even for small step heights. (C) 2014 AIP Publishing LLC.
\end{abstract}

[http://dx.doi.org/10.1063/1.4898066]

Graphene is the material with the highest thermal conductivity reported so far, ${ }^{1-3}$ with important prospective applications for example for thermal management of nanoelectronics. ${ }^{4,5}$ The ultimately thin membrane adhere well to substrates and typically will ripples, wrinkles, and bubbles form when graphene is transferred onto a flat substrate. On the other hand, since graphene is known to cling to the smallest irregularities, ${ }^{6}$ this also results in deformation and bending caused by the conformation of graphene to a irregular surface. This could, for instance, be steps in surfaces such as $\mathrm{SiC}$ or edges of other $2 \mathrm{D}$ materials such as $\mathrm{BN}$. It is thus highly relevant to consider the effects of deformation on the thermal transport properties of graphene. There are several recent studies which investigate the effects of substrate induced geometrical modulations on the electronic and transport properties of graphene. ${ }^{7-10}$ In particular, Low and coworkers ${ }^{9}$ considered the effect on the electronic transport when graphene is deformed due to physisorption on a flat substrate presenting an abrupt step. They used a simple Lennard-Jones potential to model the substrate-graphene interaction with parameters corresponding to a step in $\mathrm{SiC}$, and found that the bend itself causes an insignificant scattering of the electrons. Also, the related effect of ripples and wrinkles on electronic structure and transport in graphene on substrates has been investigated. ${ }^{11-13}$

Inspired by the study of Low et al., ${ }^{9}$ we here consider phonon transport for a model of an abrupt step in a otherwise structureless substrate. We calculate the transport for various step-heights and interaction strengths. The effects of the substrate are two-fold. First, (i) the geometry of graphene is modulated by the irregularity of the substrate, which alters the force constants locally and therefore scatters phonons. Second, (ii) the substrate gives rise to a renormalization of the vibrational modes and increases the line widths (i.e., reduces phonon lifetimes). Here, we focus on the

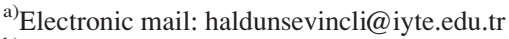

${ }^{\text {b) }}$ Electronic mail: mads.brandbyge@nanotech.dtu.dk
}

deformation (i) and neglect the dynamics of the structureless substrate.

We find that the effect of the substrate induced bend in the graphene on the phonon transport is not negligible, and can reduce the conductance with more than $10 \%$ at room temperature. For very strong substrate interaction, we obtain a decrease in phonon transmission comparable to that of a $s p^{3}$ kink-line induced by linear adsorption by hydrogen. ${ }^{10,14}$

The graphene-substrate step model geometry studied here is shown in Fig. 1(a). The substrate is treated as a static continuum with an abrupt step at $x=0$, parallel to the $y$-axis. We employ the density functional tight-binding (DFTB) method $^{15}$ to describe graphene while the van der Waals $(\mathrm{vdW})$ interaction between the sheet and the substrate is modelled with the 6-12 Lennard-Jones (LJ) potential $V_{L J}(r)=4 \epsilon_{\mathrm{LJ}}\left((\sigma / r)^{12}-(\sigma / r)^{6}\right){ }^{16,29}$ The direction transverse to the step $(y)$ is described by periodic boundary conditions using $8 \quad k_{y}$-points in the DFTB force-constant calculations. The ends of the sheet are first left free to float at a fixed distance over the substrate, in order to find the correct bending geometry. Then, the ends are connected to semiinfinite graphene sheets which will serve as reservoirs in transport calculations. The minimum-energy geometries are calculated by minimizing the forces within a tolerance of $10^{-4} \mathrm{eV} / \AA$. The force constant matrices are obtained by finite displacements of graphene atoms $\left(10^{-2} \AA\right)$ in each direction. ${ }^{17}$ An infinite mass is attributed to the substrate in order to disregard its dynamics. The phonon transmission was calculated using the Green's function method, see, e.g., Ref. 18, and averaged over $25 k_{y}$-points.

We fix the substrate graphene distance to $3.4 \AA$ $(\sigma=3.03 \AA)$ corresponding to the interlayer spacing in graphite and a typical interlayer distance for van der Waals heterolayers. The same distance is used for defining the cross sectional area for phonon transmission and conductance allowing for comparison with bulk 3D materials. We calculate the phonon transport for a variation of step heights $\left(h_{s}=1,5,10,20,50 \AA\right)$. Following Low et al. ${ }^{9}$ we use as a starting point $\epsilon_{\mathrm{LJ}}=40 \mathrm{meV}$ corresponding to a $\mathrm{SiC}$ 
(a)
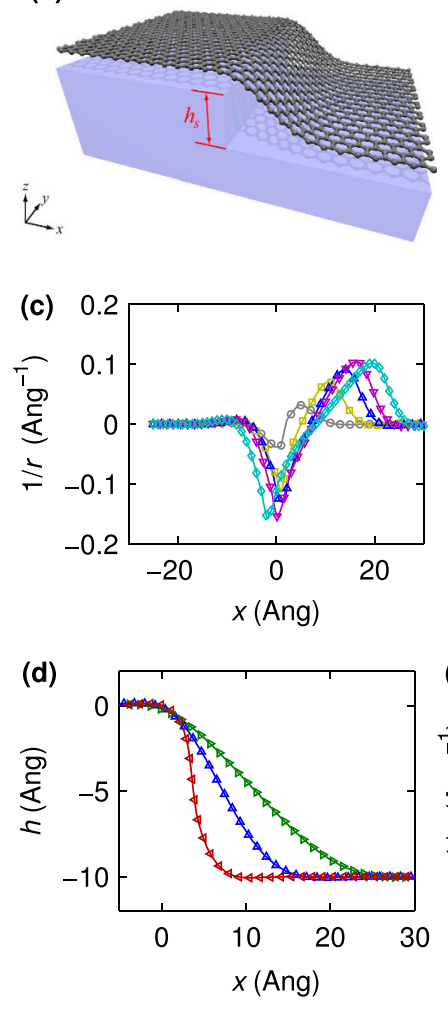

(b)
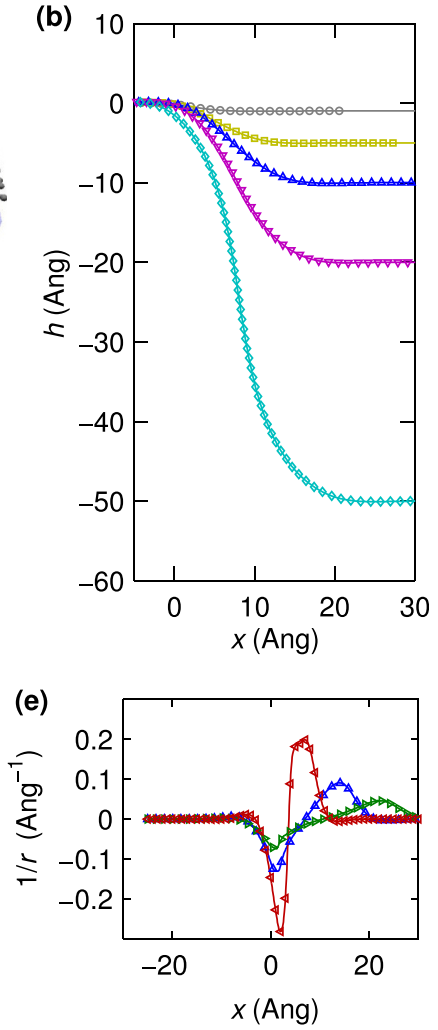

FIG. 1. (a) Illustration of graphene over a substrate step with height $h_{s}$. The step is located at $x=0$ and is parallel to the $y$-axis. The profile of the sheet on the $x z$-plane and the radius of curvature are plotted for $h_{s}=1,5,10,20$, and $50 \AA$ with $\epsilon_{L J}=40 \mathrm{meV}$ ((b) and (c)); and for $h_{s}=10 \AA$ with $\epsilon_{L J}=20$, $40,160 \mathrm{meV}((\mathrm{d})$ and $(\mathrm{e}))$.

substrate. However, adhesion energy of graphene display a large variation depending on the substrate. On insulating surfaces, the estimations for the binding energy per carbon atom ranges from 10 to $77 \mathrm{meV} .^{6,19-23}$ On metallic substrates, density functional theory suggests that it can be as high as $327 \mathrm{meV}^{24-26} \mathrm{We}$ choose $\epsilon_{\mathrm{LJ}}=20,40$, and $160 \mathrm{meV}$ with $h_{s}=10 \AA$ to address the effects of the coupling strength.

Bending profiles are plotted for various step heights with $\epsilon_{\mathrm{LJ}}=40 \mathrm{meV}$ (Fig. 1(b)) and for $h_{s}=10 \AA$ with $\epsilon_{\mathrm{LJ}}=20,40$, and $160 \mathrm{meV}$ (Fig. 1(d)), and the corresponding inverse radii of curvature $1 / r$ are plotted in Figs. 1(c) and $1(\mathrm{e})$. Varying $h_{s}$ from 1 to $50 \AA$, the minimum values of $r$ range between 25 and $6.3 \AA$ at the upper edge, and between 31.3 to $9.6 \AA$ at the lower edge, and the change in $r$ is significantly slower when $h_{s} \geq 10 \AA$. For a step height of $10 \AA$, we find that the minimum radius of curvature $r$ is 13.5, 7.7, and $3.3 \AA(21.7,10.9$, and $5 \AA)$ for $\epsilon_{\mathrm{LJ}}=20,40$, and $160 \mathrm{meV}$ at the upper (lower) edge, respectively. These values are in agreement with those in Ref. 9.

Phonon transmission spectra per cross section are shown in Fig. 2. In Fig. 2(a), we consider the transmission spectra of flat graphene for varying $\epsilon_{\mathrm{LJ}}$. The out-of-plane acoustic modes gain a gap at zero energy since the translational symmetry is broken in the $z$-direction, the size of the gap increases with the square-root of $\epsilon_{\mathrm{LJ}}$. Besides this, the substrate does not alter the transmission spectra of the flat graphene significantly. Keeping $\epsilon_{\mathrm{LJ}}$ constant at $40 \mathrm{meV}$, the effect of varying $h_{s}$ on transmission is plotted in Fig. 2(b). The effect of the step on the transmission is most pronounced (a)
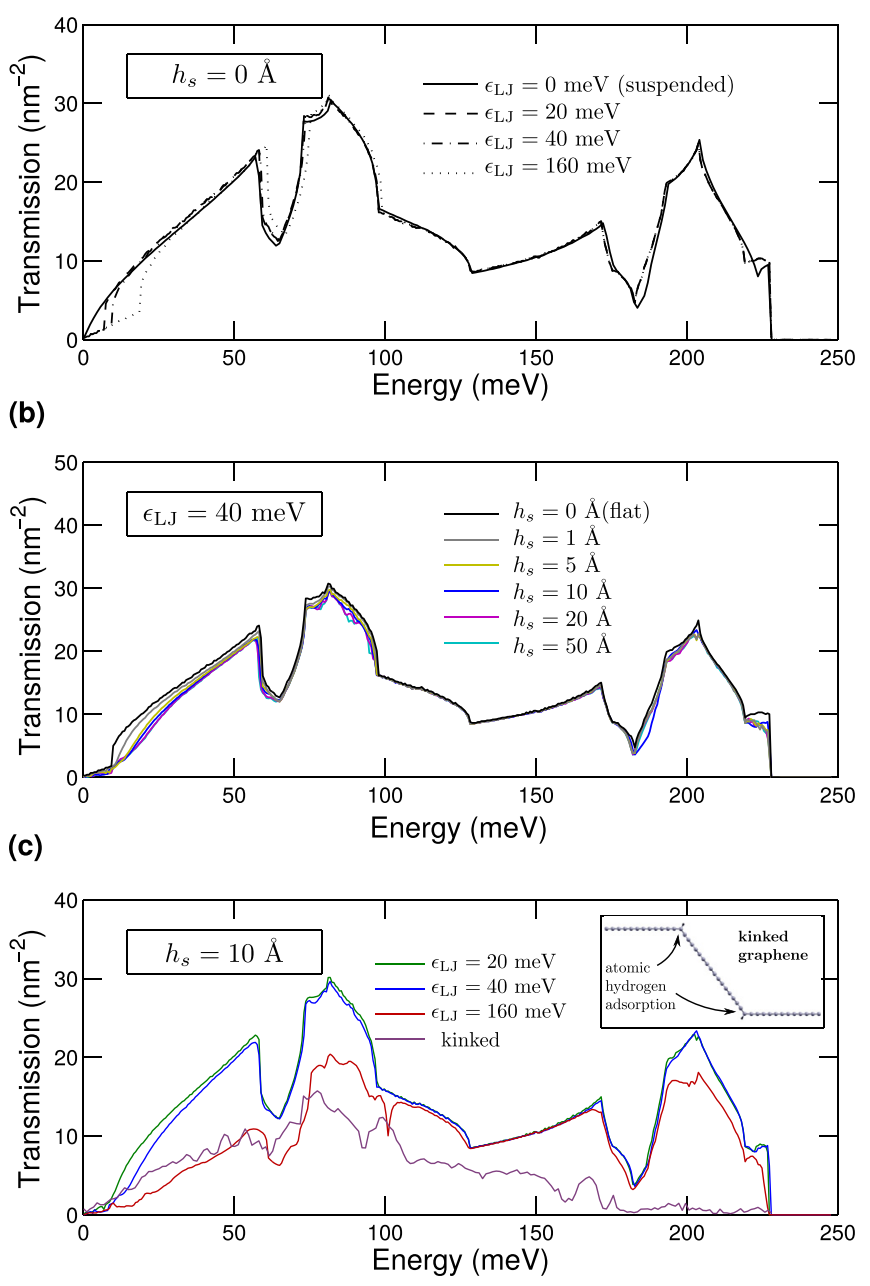

FIG. 2. Transmission per cross section area for various structures. The effect of substrate coupling in the absence of a step height for different coupling strengths are given in (a). Transmission with varying the step height, $h_{s}$, for fixed coupling strength $\epsilon_{\mathrm{LJ}}=40 \mathrm{meV}$ (b), and with varying the coupling strength for a fixed step height $h_{s}=10$ (c).

at the lower vibrational energies. As is the case for the minimum $r$, the transmission spectra are also less sensitive to $h_{s}$ when it is greater than $10 \AA$, but it is quite sensitive to the interaction strength. In Fig. 2(c), we plot transmission for $h_{s}=10 \AA$ and different $\epsilon_{\mathrm{LJ}}$, namely 20,40 , and $160 \mathrm{meV}$. The effect of the step when $\epsilon_{\mathrm{LJ}}=20 \mathrm{meV}$ is relatively small, but it is substantial for $\epsilon_{\mathrm{LJ}}=160 \mathrm{meV}$.

We also consider the effect of kinks on phonon transport. Since bending increases the chemical reactivity of graphene sheet, linear kinks can be produced by adsorption of atomic hydrogen at the step edges. ${ }^{27,28}$ Hydrogen adsorption not only modifies the hybridization but also bends the sheet abruptly with a vanishingly small radius of curvature, i.e., generates a kink, see Fig. 2(c), inset. Due to the $s p^{3}$ bonding, kinks were predicted to form efficient barriers for electron transmission. ${ }^{10}$ The case is similar for phonons. In Fig. 2(c), the phonon transmission spectrum of graphene with double kinks at the upper and lower edges of the step with $h_{s}=17 \AA$ is also plotted. The phonon transmission is severely altered due to kinks. In the case of smooth bends, transmission is mostly affected at lower energies, while in the kinked case, higher frequency phonons are also strongly suppressed. 
Thermal conductance per cross section is calculated as

$$
\kappa=\frac{k_{B}}{2 \pi} \int d \omega p(\omega, T) \mathcal{T}(\omega),
$$

with $k_{B}$ being the Boltzmann constant, $T$ temperature, and $\mathcal{T}$ the transmission per cross section. The weight function is $p=-x^{2} \partial f_{B} / \partial x$ with $x=\hbar \omega / k_{B} T$ and $f_{B}=\left(e^{x}-1\right)^{-1}$ being the Bose function. We note that $p$ has its peak value $(p=1)$ at $x=0$, and half-maximum at $x \approx 2.983$. That is, at room temperature, most of the contribution is due to phonons with $\hbar \omega<75 \mathrm{meV}$. For this reason, the substrate induced energy gap of out-of-plane modes are not affecting the room temperature properties dramatically. We find a significant reduction of conductance due to the finite step heights (Fig. 3). For $h_{s}=10 \AA$, and $\epsilon_{\mathrm{LJ}}=40 \mathrm{meV}, \kappa$ is reduced by $44 \%, 11 \%$, $8 \%$, and $7 \%$ at $50 \mathrm{~K}, 300 \mathrm{~K}, 500 \mathrm{~K}$, and $1000 \mathrm{~K}$, respectively. For $h_{s}=1 \AA(50 \AA)$, the reductions are $22 \%, 5 \%, 4 \%$, and $4 \%(47 \%, 12 \%, 10 \%$, and $9 \%)$. Depending on the interaction strength, $\kappa$ is reduced by $5 \%, 11 \%$, and $47 \%$ at room temperature for $\epsilon_{\mathrm{LJ}}=20 \mathrm{meV}, 40 \mathrm{meV}$, and $160 \mathrm{meV}$, respectively.

In Fig. 4(a), we plot the ratio of thermal conductance of graphene over a step to that of flat graphene with

(a)

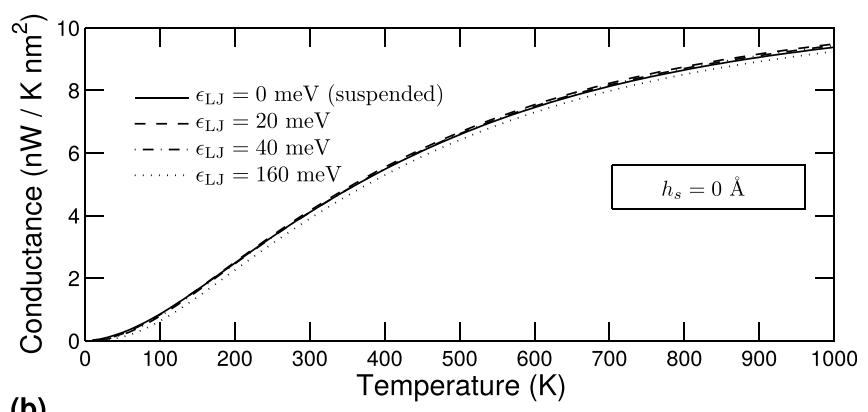

(b)

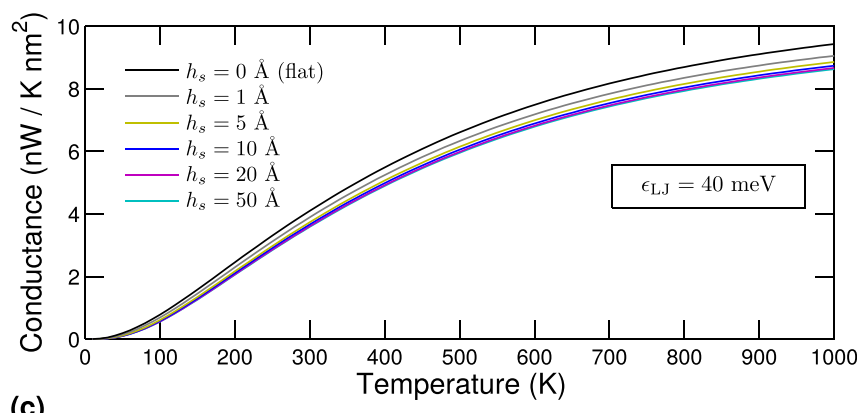

(c)

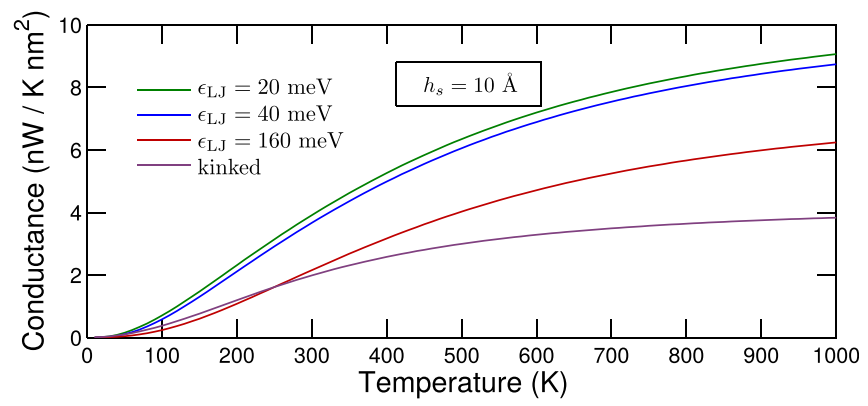

FIG. 3. Thermal conductance per cross section area (a) for flat graphene with different strengths of substrate interaction, (b) for different step heights with a fixed interaction strength $\left(\epsilon_{\mathrm{LJ}}=40 \mathrm{meV}\right)$, (c) for different interaction strengths and a fixed step height $\left(h_{s}=10 \AA\right)$. Thermal conductance of kinked graphene is also shown for comparison. (a)

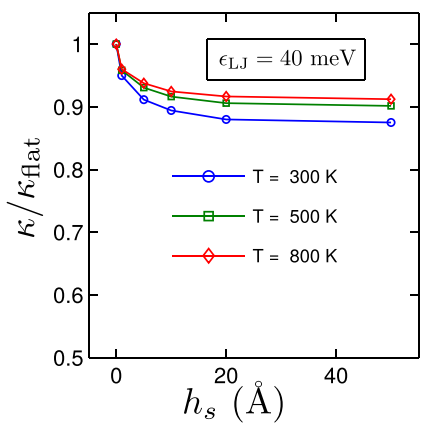

(b)

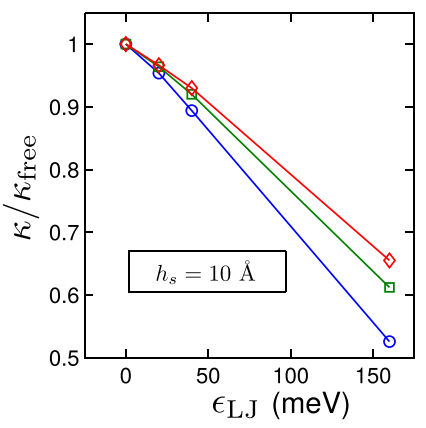

FIG. 4. The ratio of thermal conductance of graphene over a step for a fixed interaction strength $\left(\epsilon_{\mathrm{LJ}}=40 \mathrm{meV}\right)$ as a function of the step height (a), and for a fixed step height $\left(h_{s}=10 \AA\right)$ as a function of the interaction strength (b).

$\epsilon_{\mathrm{LJ}}=40 \mathrm{meV}$. Even a small step of $1 \AA$ reduces $\kappa$ by approximately $5 \%$, while the reduction saturates at around $10 \%$ for higher steps. On the other hand, keeping $h_{s}=10 \AA$, the ratio of $\kappa$ to that of free standing graphene is sensitive to the coupling strength as seen in Fig. 4(b). The thermal resistance of a step can be defined as $R_{s}=\kappa^{-1}-\kappa_{\text {flat }}^{-1}$. At $300 \mathrm{~K}, R_{S}=$ 0.013 (0.035) $\mathrm{nm}^{2} \mathrm{~K} / \mathrm{nW}$ for $h_{s}=1$ (50) $\AA$ and $\epsilon_{\mathrm{LJ}}=40 \mathrm{meV}$, where $\kappa_{\text {flat }}^{-1}=0.243 \mathrm{~nm}^{2} \mathrm{~K} / \mathrm{nW}$. Assuming that resistance due to individual steps are additive, one concludes that only a small number of substrate steps can reduce the thermal conductivity by a substantial amount and thus play a major role for thermal transport in graphene when supported by a substrate.

We thank Peter Bøggild and Jesper T. Rasmussen for fruitful discussions. H.S. acknowledges support from Danish Council for Independent Research, Individual Postdoc Grant (No. 0602-02477B-FTP), from Scientific and Research Council of Turkey, TÜBİTAK (BIDEB-113C032), and from Bilim Akademisi-the Science Academy, Turkey under the BAGEP program. We also would like to thank TÜBITAK ULAKBIM High Performance Computing Center.

${ }^{1}$ A. A. Balandin, S. Ghosh, W. Bao, I. Calizo, D. Teweldebrhan, F. Miao, and C. N. Lau, Nano Lett. 8, 902 (2008).

${ }^{2}$ A. Balandin, Nat. Mater. 10, 569 (2011).

${ }^{3}$ D. L. Nika and A. A. Balandin, J. Phys.: Condens. Matter 24, 233203 (2012).

${ }^{4}$ S. Ghosh, I. Calizo, D. Teweldebrhan, E. P. Pokatilov, D. L. Nika, A. A. Balandin, W. Bao, F. Miao, and C. N. Lau, Appl. Phys. Lett. 92, 151911 (2008).

${ }^{5}$ K. M. F. Shahil and A. A. Balandin, Solid State Commun. 152, 1331 (2012).

${ }^{6} \mathrm{~J}$. S. Bunch and M. L. Dunn, Solid State Commun. 152, 1359 (2012).

${ }^{7}$ J.-K. Lee, S. Yamazaki, H. Yun, J. Park, G. P. Kennedy, G.-T. Kim, O. Pietzsch, R. Wiesendanger, S. Lee, S. Hong, U. Dettlaff-Weglikowska, and S. Roth, Nano Lett. 13, 3494 (2013).

${ }^{8}$ J. Hicks, A. Tejeda, A. Taleb-Ibrahimi, M. S. Nevius, F. Wang, K. Shepperd, J. Palmer, F. Bertran, P. Le Fèvre, J. Kunc, W. A. de Heer, C. Berger, and E. H. Conrad, Nat. Phys. 9, 49 (2013).

${ }^{9}$ T. Low, V. Perebeinos, J. Tersoff, and P. Avouris, Phys. Rev. Lett. 108, 096601 (2012).

${ }^{10}$ J. T. Rasmussen, T. Gunst, P. Bøggild, A.-P. Jauho, and M. Brandbyge, Beilstein J. Nanotechnol. 4, 103 (2013).

${ }^{11}$ W. Bao, F. Miao, Z. Chen, H. Zhang, W. Jang, C. Dames, and C. N. Lau, Nat. Nanotechnol. 4, 562 (2009).

${ }^{12}$ R. Miranda and A. L. Vázquez de Parga, Nat. Nanotechnol. 4, 549 (2009).

${ }^{13}$ Z. Pan, N. Liu, L. Fu, and Z. Liu, J. Am. Chem. Soc. 133, 17578 (2011). 
${ }^{14}$ L. A. Chernozatonskii, P. B. Sorokin, and J. W. Brüning, Appl. Phys. Lett. 91, 183103 (2007).

${ }^{15}$ B. Aradi, B. Hourahine, and T. Frauenheim, J. Phys. Chem. A 111, 5678 (2007).

${ }^{16}$ There are other potentials available for modelling inter-layer interactions, like the spherically symmetric inter-atomic potential used in Ref. 29. Since the scope of the present study is the effects of a generic substrate, we limit our discussion to Lennard-Jones potential only.

${ }^{17}$ A. Togo, F. Oba, and I. Tanaka, Phys. Rev. B 78, 134106 (2008).

${ }^{18}$ H. Sevinçli, C. Sevik, T. Çağin, and G. Cuniberti, Sci. Rep. 3, 1228 (2013).

${ }^{19}$ A. Mattausch and O. Pankratov, Phys. Rev. Lett. 99, 076802 (2007).

${ }^{20}$ M. Z. Hossain, Appl. Phys. Lett. 95, 143125 (2009).

${ }^{21}$ Z. Ao, M. Jiang, Z. Wen, and S. Li, Nanoscale Res. Lett. 7, 158 (2012).

${ }^{22}$ Y.-J. Kang, J. Kang, and K. J. Chang, Phys. Rev. B 78, 115404 (2008).
${ }^{23}$ T. C. Nguyen, M. Otani, and S. Okada, Phys. Rev. Lett. 106, 106801 (2011).

${ }^{24}$ T. Yoon, W. C. Shin, T. Y. Kim, J. H. Mun, T.-S. Kim, and B. J. Cho, Nano Lett. 12, 1448 (2012).

${ }^{25}$ P. A. Khomyakov, G. Giovannetti, P. C. Rusu, G. Brocks, J. van den Brink, and P. J. Kelly, Phys. Rev. B 79, 195425 (2009).

${ }^{26}$ M. Vanin, J. J. Mortensen, A. K. Kelkkanen, J. M. Garcia-Lastra, K. S. Thygesen, and K. W. Jacobsen, Phys. Rev. B 81, 081408 (2010).

${ }^{27}$ D. Srivastava, D. W. Brenner, J. D. Schall, K. D. Ausman, M. Yu, and R. S. Ruoff, J. Phys. Chem. B 103, 4330 (1999).

${ }^{28}$ D. C. Elias, R. R. Nair, T. M. G. Mohiuddin, S. V. Morozov, P. Blake, M. P. Halsall, A. C. Ferrari, D. W. Boukhvalov, M. I. Katsnelson, A. K. Geim, and K. S. Novoselov, Science 323, 610 (2009).

${ }^{29}$ D. L. Nika, A. I. Cocemasov, and A. A. Balandin, Appl. Phys. Lett. 105, 031904 (2014). 